\title{
Radio Signal by Galactic Dark Matter
}

\author{
E. Borriello ${ }^{a}$, A. Cuoco $^{b}$, G. Miele ${ }^{\mathrm{a}} \mathrm{c} *$ \\ a Università di Napoli "Federico II", Dipartimento di Scienze Fisiche and INFN - Sezione di Napoli, \\ Complesso Universitario di Monte S.Angelo, Via Cithia, 80126, Napoli, Italy \\ b Department of Physics and Astronomy, University of Aarhus, Ny Munkegade, Bygn. 15208000 Aarhus \\ Denmark \\ ' Instituto de Física Corpuscular (CSIC-Universitat de València), Ed. Institutos de Investigación, \\ Apartado de Correos 22085, E-46071 València, Spain.
}

An interesting strategy for indirect detection of Dark Matter comes through the amounts of electrons and positrons usually emitted by DM pair annihilation. The $e^{+} e^{-}$gyrating in the galactic magnetic field then produce secondary synchrotron radiation. The radio emission from the galactic halo as well as from its expected substructures if compared with the measured diffuse radio background can provide constraints on the physics of WIMPs. In particular one gets the bound of $\left\langle\sigma_{A} v\right\rangle=10^{-24} \mathrm{~cm}^{3} \mathrm{~s}^{-1}$ for a DM mass $m_{\chi}=100 \mathrm{GeV}$ even though sensibly depending on the astrophysical uncertainties.

\section{Introduction}

Among the indirect DM detection channels, the radio emission due to secondary electrons or positrons can represent a chance to look for DM annihilation. During the process of thermalization in the galactic medium the high energy $e^{+}$ and $e^{-}$release secondary low energy radiation, in particular in the radio and X-ray band, which in principle could be detected. Furthermore, while the astrophysical uncertainties affecting this signal are similar to the case of direct $e^{+}, e^{-}$detection, the sensitivities are quite different, and, in particular, the radio band allows for a the discrimination of tiny signals even in a background many order of magnitudes more intense.

Indirect detection of DM annihilation through secondary photons has received recently an increasing attention, exploring the expected signature in X-rays [1-3], at radio wavelengths [4-7], or both [8-10]. In this paper we will focus our analysis on the radio signal expected from the Milky Way (MW) halo and its substructures. The results and the details of the following approach can be found in Ref. [11].

*Presented by G. Miele

\section{Dark matter distribution}

Our knowledge of the DM spatial distribution on galactic and subgalactic scales has greatly improved thanks to recent high resolution zoomed N-body simulations [15-18]. These simulations indicate that for the radial profile of the galactic halo the usual Navarro-Frank-White (NFW) distribution [19]

$\rho(r)=\frac{\rho_{h}}{\frac{r}{r_{h}}\left(1+\frac{r}{r_{h}}\right)^{2}}$,

still works as a good approximation over all the resolved scales. The NFW profile is in fair agreement with the observed Milky Way rotation curve [20], although, depending on the employed model, it is possible to find an agreement for many different DM profiles. We emphasize, however, that the various profiles differ mainly in the halo center (for $r \lesssim 1 \mathrm{kpc}$ ) where the uncertainties, both in numerical simulations and from astrophysical observations are maximal. Thus, our analysis which explicitly excludes the galactic center, does not crucially depend on the choice of the profile.

The parameters describing the halo are then determined imposing the DM density to be equal to $\rho_{S}=0.365 \mathrm{GeV} c^{-2} \mathrm{~cm}^{-3}$ near the Solar Sys- 
tem, at a galactocentric distance of $R_{S}=8.5 \mathrm{kpc}$.

Simulations, however, predict a DM distribution sum of a smooth halo component, and of an additional clumpy one with total masses roughly of the same order of magnitude. For the subhalo population we will assume a mass distribution $\propto m_{c l}^{-2}$ and that they are spatially distributed following the NFW profile of the main halo. The mass spectrum number density of subhaloes, in galactocentric coordinates $\vec{r}$, is thus given by

$\frac{\mathrm{d} n_{c l}}{\mathrm{~d} m_{c l}}\left(m_{c l}, \vec{r}\right)=A\left(\frac{m_{c l}}{M_{c l}}\right)^{-2}\left(\frac{r}{r_{h}}\right)^{-1}\left(1+\frac{r}{r_{h}}\right)^{-2}$,

where $A$ is a dimensional normalization constant.

By using the constraints described in [11] one can fix the values of free parameters $r_{h}, \rho_{h}$ and $A$, hence obtaining $r_{h}=14.0 \mathrm{kpc}, \rho_{h}=0.572 \mathrm{GeV}$ $c^{-2} \mathrm{~cm}^{-3}$ and $A=1.16 \times 10^{-19} \mathrm{kpc}^{-3} \mathrm{M}_{\odot}^{-1}$.

A further piece of information is required to derive the annihilation signal from the clumps, namely how the DM is distributed inside the clumps themselves. We will assume that each clump follows a NFW profile whose parameters are fixed assuming the clumps concentration according to [21].

\section{Radio Data}

In the following we will derive constraints on the DM emission comparing the expected diffuse emission from the smooth halo and the unresolved population of clumps with all sky observation in the radio band. In the frequency range 100 MHz-100 GHz where the DM synchrotron signal is expected, various astrophysical processes contribute to the observed diffuse emission. Competing synchrotron emission is given by Cosmic Ray electrons accelerated in supernovae shocks dominating the radio sky up to $\sim 10 \mathrm{GHz}$. At higher frequencies the Cosmic Microwave Background $(\mathrm{CMB})$ and its anisotropies represent the main signal. However, thanks to the very sensitive multi-frequency survey by the WMAP satellite, this signal (which represents thus a background for DM searches) can be modeled in a detailed way and can thus be removed from the observed radio galactic emission [22]. Other processes contributing in the $10-100 \mathrm{GHz}$ range are given by thermal bremsstrahlung (free-free emission) of electrons on the galactic ionized gas, and emission by small grains of vibrating or spinning dust.

In the following our approach will be to compare the DM signal with the observed radio emission where only the CMB is modeled and removed. For this purpose we use the code described in [23] where most of the radio survey observations in the range $10 \mathrm{MHz}-100 \mathrm{GHz}$ are collected and a scheme to derive interpolated, $\mathrm{CMB}$ cleaned sky maps at any frequency in this range is described.

\section{DM Synchrotron Signal}

In a standard scenario where WIMPs experience a non exotic thermal history, a typical mass range for these particles is $50 \mathrm{GeV} \lesssim m_{\chi} \lesssim 1 \mathrm{TeV}$, while a simple estimate for their (thermally averaged) annihilation cross section yields $\left\langle\sigma_{A} v\right\rangle=3 \times 10^{-27} \mathrm{~cm}^{3} \mathrm{~s}^{-1} / \Omega_{c d m} h^{2}$, giving $\left\langle\sigma_{A} v\right\rangle \approx 3 \times 10^{-26} \mathrm{~cm}^{3} \mathrm{~s}^{-1}$ for $\Omega_{c d m} h^{2} \approx 0.1$ as resulting from the latest WMAP measurements. However, this naive relation can fail badly if, for example, coannihilations play a role in the WIMP thermalization process [24], and a much wider range of cross sections should be considered viable. In this work we consider values of $m_{\chi}$ from about $10 \mathrm{GeV}$ to about $1 \mathrm{TeV}$, and $\left\langle\sigma_{A} v\right\rangle$ in the range $10^{-26} \div 10^{-21} \mathrm{~cm}^{3} \mathrm{~s}^{-1}$

The $e^{+} e^{-} \quad$ annihilation spectrum, $\mathrm{d} N_{e} / \mathrm{d} E_{e}\left(E_{e}\right)$, for a given super-symmetric WIMP candidate can be calculated for example with the DarkSUSY package [25]. Once injected in the galaxy, by neglecting diffusion (see Ref. [11] for details), the emitted $e^{+} e^{-}$follow the steady state distribution which reads

$\frac{\mathrm{d} n_{e}}{\mathrm{~d} E_{e}}\left(E_{e}, \vec{r}\right)=\frac{\tau(\vec{r})}{E_{e}} \int_{E_{e}}^{m_{\chi} c^{2}} d E_{e}^{\prime} Q\left(E_{e}^{\prime}, r\right)$,

where

$Q\left(E_{e}, r\right)=\frac{1}{2}\left(\frac{\rho(r)}{m_{\chi}}\right)^{2}\left\langle\sigma_{A} v\right\rangle \frac{\mathrm{d} N_{e}}{\mathrm{~d} E_{e}}\left(E_{e}\right)$

is the constant rate at which DM annihilation injects new electrons in the galaxy and $\tau=$ $E_{e} / b\left(E_{e}, \vec{r}\right)$ is the cooling time, resulting from the 
sum of several energy loss processes that affect electrons. In the following we will consider synchrotron emission and Inverse Compton Scattering (ICS) off the background photons (CMB and starlight) only, which are the faster processes and thus the ones really driving the electrons equilibrium. Other processes, like synchrotron self absorption, ICS off the synchrotron photons, $e^{+} e^{-}$ annihilation, Coulomb scattering over the galactic gas and bremsstrahlung are generally slower. They can become relevant for extremely intense magnetic field, possibly present in the inner parsecs of the galaxy [5], and thus will be neglected in this analysis.

The synchrotron spectrum of an electron gyrating in a magnetic field has its prominent peak at the resonance frequency

$\nu=3.7\left(\frac{B}{\mu \mathrm{G}}\right)\left(\frac{E_{e}}{\mathrm{GeV}}\right)^{2} \mathrm{MHz}$.

This implies that, in practice, a $\delta$-approximation around the peaks works extremely well. Using this frequency peak approximation, the synchrotron emissivity can be defined as

$j_{\nu}(\nu, \vec{r})=\frac{\mathrm{d} n_{e}}{\mathrm{~d} E_{e}}\left(E_{e}(\nu), \vec{r}\right) \frac{\mathrm{d} E_{e}(\nu)}{\mathrm{d} \nu} b_{s y n}\left(E_{e}(\nu), \vec{r}\right)$.

This quantity is then integrated along the line of sight to get the final synchrotron flux across the sky:

$\frac{\mathrm{d}^{2} I_{\nu}}{\mathrm{d} l \mathrm{~d} b}=\frac{\cos b}{4 \pi} \int_{0}^{\infty} j_{\nu} \mathrm{d} s$,

where $(l, b)$ are coordinates on the sphere and $s$ the line of sight coordinate.

The two contributes mainly differ due to the fact that, while the halo emission is proportional to the square of the NFW profile, the clumps one is simply proportional to it. (see Ref. [11]).

The halo component dominates in the central region of the galaxy, inside a disk with a radius of about 30 degrees, while the clumps component represents the main contribution in the external region (see fig. 2).

\section{DM Annihilation constraints}

The pattern and intensity of the DM radio map resulting from the sum of the contributions

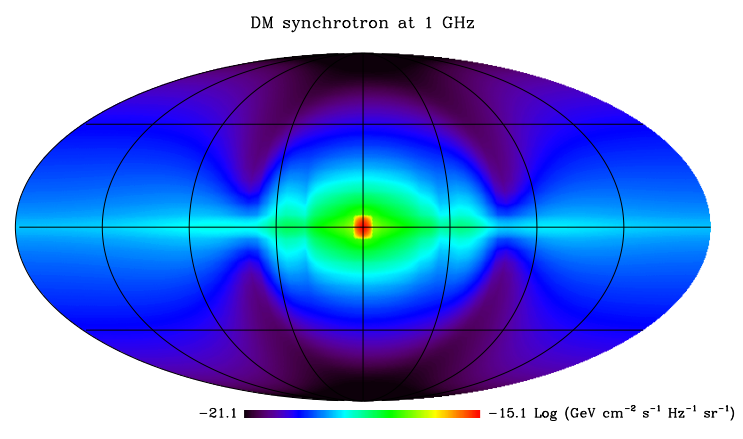

Figure 1. Sky map of the galactic radio signal generated by the DM smooth halo and unresolved clumps at the frequency of $1 \mathrm{GHz}$ for $m_{\chi}=100$ $\mathrm{GeV}$ and $\left\langle\sigma_{A} v\right\rangle=3 \times 10^{-26} \mathrm{~cm}^{3} \mathrm{~s}^{-1}$. The peculiar shape of the signal, pinched approximately at $\pm 30^{\circ}$ and $\pm 60^{\circ}$, reflects basically the structure of the magnetic field as seen in projection from the Solar System, where the observer is located.

from the smooth halo and unresolved clumps is shown in Fig. 1 for $m_{\chi}=100 \mathrm{GeV}$ and $\left\langle\sigma_{A} v\right\rangle=$ $3 \times 10^{-26} \mathrm{~cm}^{3} \mathrm{~s}^{-1}$. Similar maps are obtained at different frequencies and different $m_{\chi}$ and $\left\langle\sigma_{A} v\right\rangle$ to obtain DM exclusion plots. For our analysis we use a small mask covering a $15^{\circ} \times 15^{\circ}$ region around the galactic center where energy loss processes other than synchrotron and ICS start possibly to be relevant. We include the galactic plane although this region has basically no influence for the constraints on the DM signal.

In Fig. 3 we show the radio constraints on the $\mathrm{DM}$ annihilation signal in the $m_{\chi}-\left\langle\sigma_{A} v\right\rangle$ plane for various frequencies and various choices of the foreground. Several comments are in order. First, we can see that, as expected, the use of the haze at 23 $\mathrm{GHz}$ gives about one order of magnitude better constraints with respect to the synchrotron foregrounds at the same frequency. However, using also the information at other frequencies almost the same constraints can be achieved. This information in particular is complementary giving better constraints at lower DM masses. This is easily understood since a smaller DM mass increases 


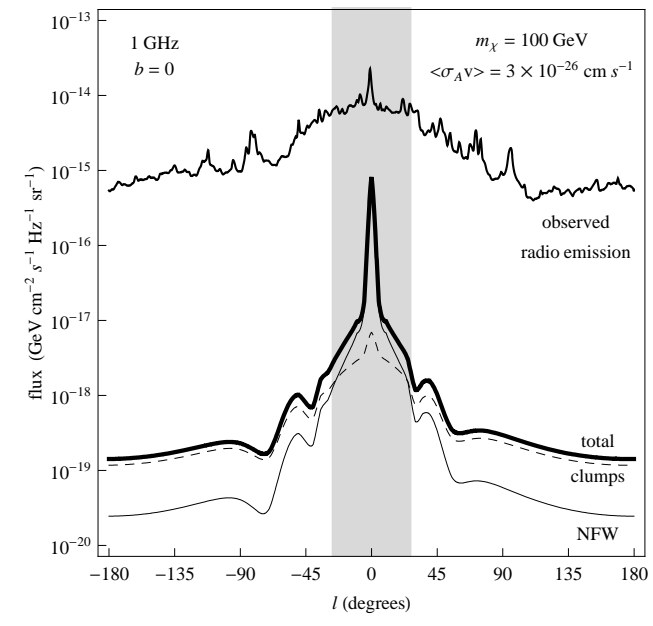

Figure 2. DM synchrotron profile for the halo and unresolved substructures and their sum at $1 \mathrm{GHz}$ for $m_{\chi}=100 \mathrm{GeV}$ and $\left\langle\sigma_{A} v\right\rangle=3 \times 10^{-26} \mathrm{~cm}^{3} \mathrm{~s}^{-1}$. The astrophysical observed emission at the same frequency is also shown. The gray band indicates the angular region within which the DM signal from the host halo dominates over the signal from substructures modeled as in section 2 .

the annihilation signal $\left(\propto m_{\chi}^{-2}\right)$ at smaller energies, and thus smaller synchrotron frequencies. In particular, the constraints improve of about one order of magnitude at $m_{\chi} \sim 100 \mathrm{GeV}$ from 23 $\mathrm{GHz}$ to $1 \mathrm{GHz}$ while only a modest improvement is achieved considering further lower frequencies as $0.1 \mathrm{GHz}$. This saturation of the constraints is due to the frequency dependence of the DM signal, that below $1 \mathrm{GHz}$ becomes flatter than the astrophysical backgrounds so that the fraction of contribution from DM is maximal at about $1 \mathrm{GHz}$.

For low masses the constraints come more and more from lower frequencies. For example for a WIMP of $30 \mathrm{GeV}$ the data at $100 \mathrm{MHz}$ are 2 orders of magnitude more constraining than the data at $10 \mathrm{GHz}$. For a WIMP of $1 \mathrm{GeV}$, from Eq.5 with a magnetic field of $\mathcal{O}(\mu \mathrm{G})$ only frequencies $\lesssim 10 \mathrm{MHz}$ would be useful to place constraints on the DM signal. Although observations at this frequency exist [23], in general the survey sky coverage is quite incomplete and the data quality is non-optimal. Observations in this very low frequency range should substantially improve with the next generation radio arrays LOFAR and SKA.

\section{Summary and conclusions}

Using conservative assumptions for the DM distribution in our galaxy we derive the expected secondary radiation due to synchrotron emission from high energy electrons produced in DM annihilation. The signal from single bright clumps offers only poor sensitivities because of diffusion effects which spread the electrons over large areas diluting the radio signal. The diffuse signal from the halo and the unresolved clumps is instead relevant and can be compared to the radio astrophysical background to derive constraints on the DM mass and annihilation cross section.

Constraints in the radio band, in particular, are complementary to similar (less stringent but less model dependent) constraints in the Xray/gamma band $[26,27]$ and from neutrinos [28]. Radio data, in particular, are more sensitive in the $\mathrm{GeV}-\mathrm{TeV}$ region while neutrinos provide more stringent bounds for very high DM masses ( $\gtrsim 10$ $\mathrm{TeV}$ ). Gammas, instead, are more constraining for $m_{\chi} \lesssim 1 \mathrm{GeV}$. The combination of the various observations provides thus interesting constraints over a wide range of masses pushing the allowed window significantly near the thermal relic possibility.

More into details, we obtain conservative constraints at the level of $\left\langle\sigma_{A} v\right\rangle \sim 10^{-23} \mathrm{~cm}^{3} \mathrm{~s}^{-1}$ for a DM mass $m_{\chi}=100 \mathrm{GeV}$ from the WMAP Haze at $23 \mathrm{GHz}$. However, depending on the astrophysical uncertainties, in particular on the assumption on the galactic magnetic field model, constraints as strong as $\left\langle\sigma_{A} v\right\rangle \sim 10^{-25} \mathrm{~cm}^{3} \mathrm{~s}^{-1}$ can be achieved. Complementary to other works which employ the WMAP Haze at $23 \mathrm{GHz}$, we also use the information in a wide frequency band in the range $100 \mathrm{MHz}-100 \mathrm{GHz}$. Adding this information the constraints become of the order of $\left\langle\sigma_{A} v\right\rangle \sim 10^{-24} \mathrm{~cm}^{3} \mathrm{~s}^{-1}$ for a DM mass $m_{\chi}=100 \mathrm{GeV}$. The multi-frequency approach 

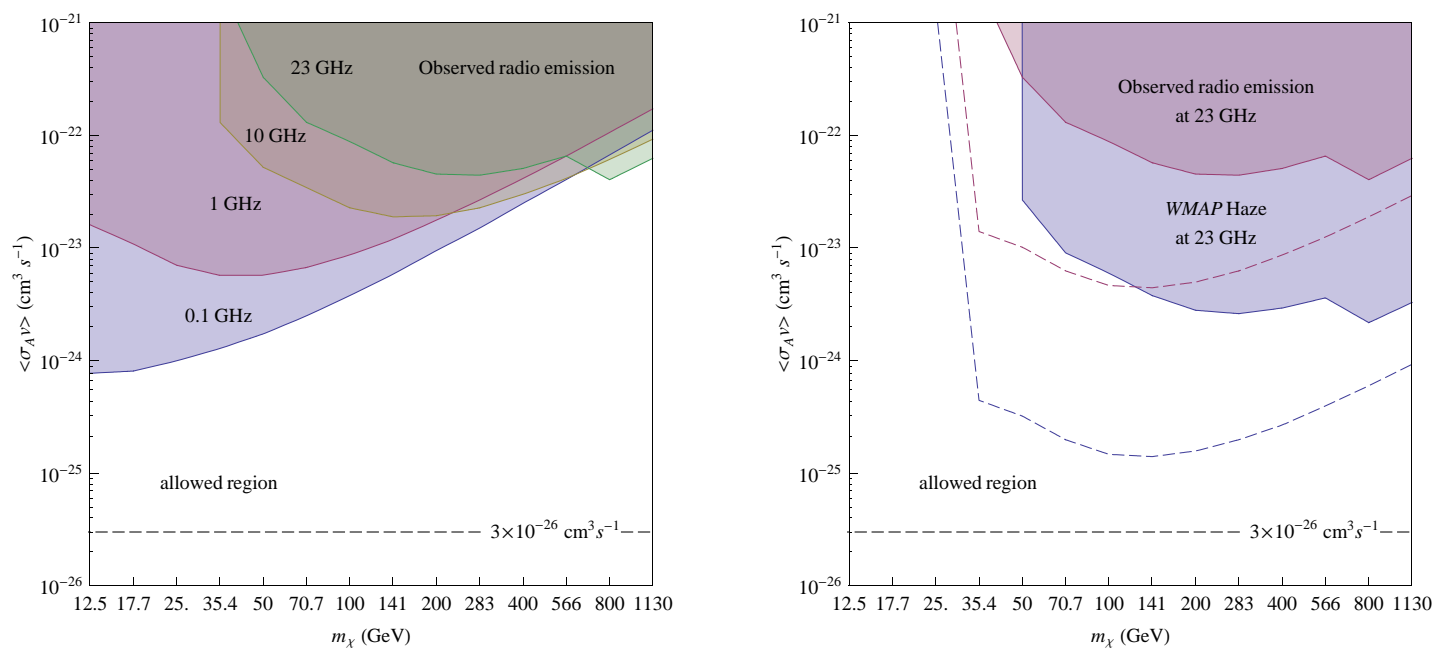

Figure 3. (Left) Constraints in the $m_{\chi}-\left\langle\sigma_{A} v\right\rangle$ plane for various frequencies without assuming synchrotron foreground removal. (Right) Constraints from the WMAP $23 \mathrm{GHz}$ foreground map and 23 $\mathrm{GHz}$ foreground-cleaned residual map (the WMAP Haze) for the TT model of magnetic field (filled regions) and for a uniform $10 \mu \mathrm{G}$ field (dashed lines).

thus gives comparable constraints with respect to the WMAP Haze only, or generally better for $m_{\chi} \lesssim 100 \mathrm{GeV}$ where the best sensitivity is achieved at $\sim \mathrm{GHz}$ frequencies.

The derived constraints are quite conservative because no attempt to model the astrophysical background is made differently from the case of the WMAP Haze. Indeed, the Haze residual map itself should be interpreted with some caution, given that the significance of the feature is at the moment still debated and complementary analyses from different groups (as the WMAP one) miss in finding a clear evidence of the feature. Definitely the multi-frequency approach will be necessary to test in a convincing way a possible DM signal like the claim related to the WMAP Haze. Progresses are expected with the forthcoming data at high frequencies from Planck and at low frequencies from LOFAR and, in a more distant future, from SKA. These surveys will help in disentangling the various astrophysical contributions thus assessing the real significance of the Haze feature. Further, the low frequency data in particular, will help to improve our knowledge of the galactic magnetic field. Progresses in these fields will provide a major improvement for the interpretation of the DM-radio connection.

\section{Acknowledgments}

G.M. acknowledges supports by the Spanish MICINN (grants SAB2006-0171 and FPA200501269) and by INFN-I.S.Fa51 and PRIN 2006 "Fisica Astroparticellare: Neutrini ed Universo Primordiale" of Italian MIUR.

\section{REFERENCES}

1. L. Bergstrom, M. Fairbairn and L. Pieri, Phys. Rev. D 74, 123515 (2006) [arXiv:astro$\mathrm{ph} / 0607327$.

2. M. Regis and P. Ullio, Phys. Rev. D 78 (2008) 043505 [arXiv:0802.0234 [hep-ph]].

3. T. E. Jeltema and S. Profumo, arXiv:0805.1054 [astro-ph].

4. P. Blasi, A. V. Olinto and C. Tyler, As- 
tropart. Phys. 18 (2003) 649 [arXiv:astro$\mathrm{ph} / 0202049]$.

5. R. Aloisio, P. Blasi and A. V. Olinto, JCAP 0405 (2004) 007 [arXiv:astro-ph/0402588].

6. A. Tasitsiomi, J. M. Siegal-Gaskins and A. V. Olinto, Astropart. Phys. 21 (2004) 637 [arXiv:astro-ph/0307375].

7. L. Zhang and G. Sigl, arXiv:0807.3429 [astro$\mathrm{ph}$.

8. S. Colafrancesco, S. Profumo and P. Ullio, Astron. Astrophys. 455 (2006) 21 [arXiv:astro$\mathrm{ph} / 0507575]$.

9. S. Colafrancesco, S. Profumo and P. Ullio, Phys. Rev. D 75 (2007) 023513 [arXiv:astro$\mathrm{ph} / 0607073]$.

10. E. A. Baltz and L. Wai, Phys. Rev. D 70 (2004) 023512 [arXiv:astro-ph/0403528].

11. E. Borriello, A. Cuoco and G. Miele, arXiv:0809.2990 [astro-ph].

12. D. Hooper, D. P. Finkbeiner and G. Dobler, Phys. Rev. D 76 (2007) 083012 [arXiv:0705.3655 [astro-ph]].

13. D. Hooper, Phys. Rev. D 77 (2008) 123523 [arXiv:0801.4378 [hep-ph]].

14. P. Grajek, G. Kane, D. J. Phalen, A. Pierce and S. Watson, [arXiv:0807.1508 [hep-ph]].

15. J. Diemand, B. Moore and J. Stadel, Nature 433 (2005) 389 [arXiv:astro-ph/0501589].

16. J. Diemand, M. Kuhlen and P. Madau, Astrophys. J. 657 (2007) 262 [arXiv:astro$\mathrm{ph} / 0611370]$.

17. M. Kuhlen, J. Diemand and P. Madau, arXiv:0805.4416 [astro-ph].

18. V. Springel et al., arXiv:0809.0898 [astro-ph].

19. J. F. Navarro, C. S. Frenk and S. D. M. White, Astrophys. J. 490 (1997) 493 [arXiv:astro-ph/9611107].

20. A. Klypin, H. Zhao and R. S. Somerville, Astrophys. J. 573 (2002) 597 [arXiv:astro$\mathrm{ph} / 0110390]$.

21. J. S. Bullock et al., Mon. Not. Roy. Astron. Soc. 321 (2001) 559 [arXiv:astro$\mathrm{ph} / 9908159]$.

22. M. Tegmark, A. de Oliveira-Costa and A. Hamilton, Phys. Rev. D 68 (2003) 123523 [arXiv:astro-ph/0302496].

23. A. de Oliveira-Costa, M. Tegmark, B. M. Gaensler, J. Jonas, T. L. Landecker and P. Reich, arXiv:0802.1525 [astro-ph].

24. K. Griest and D. Seckel, Phys. Rev. D 43 (1991) 3191.

25. P. Gondolo, J. Edsjo, P. Ullio, L. Bergstrom, M. Schelke and E. A. Baltz, JCAP 0407 (2004) 008 [arXiv:astro-ph/0406204].

26. G. D. Mack, T. D. Jacques, J. F. Beacom, N. F. Bell and H. Yuksel, arXiv:0803.0157 [astro-ph].

27. M. Kachelriess and P. D. Serpico, Phys. Rev. D 76 (2007) 063516 [arXiv:0707.0209 [hep$\mathrm{ph}]$.

28. H. Yuksel, S. Horiuchi, J. F. Beacom and S. Ando, Phys. Rev. D 76 (2007) 123506 [arXiv:0707.0196 [astro-ph]]. 